\title{
Plastic upcycling
}

\author{
Plastic waste is a serious matter of concern due to its disruptive impact on the environment. While disposal \\ and reclaim strategies represent the first lines of intervention to solve this problem, upcycling options based on \\ catalytic transformations will eventually be necessary to reconvert enormous quantities of such material.
}

ecent estimates suggest that 4.8 to 12.7 million metric tonnes of plastic may have entered the ocean in $2010^{1}$. Only part of such waste can be tracked within the ocean's garbage patches or on coastal areas in the form of buoyant plastic objects. In fact, a large fraction of such waste has a tendency to degrade and settle below the sea surface layer, where it can be further ingested by marine organisms and finally accumulates in the ecosystem ${ }^{2}$. The related environmental and health threats are well known.

While the use of plastic is integral to modernity and has brought enormous benefits to different sectors including food packaging, medical devices, and telecommunications, it is obvious that current disposal and end-of-life management strategies are no longer sustainable. In order to provide a solution, a number of commendable initiatives have been kicked off, at least when it comes to plastic accumulated within seas and waterways. A few days ago, for instance, the Ocean Cleanup - a private environmental organization - announced the intention to deploy automated plastic collection units in heavily polluted rivers following successful trials in Indonesia and Malaysia. The organization has claimed a removal capacity per unit of $50,000 \mathrm{~kg}$ per day so far, complementing its ongoing efforts to reclaim plastic waste from the oceans via specialized collection systems. And this is just one of a number of efforts targeting plastic waste removal. In fact, different technologies are under investigation - see for instance the Great Bubble Barrier - but also different water environments prone to plastic accumulation are being targeted, like marinas (for example, the Seabin Project). Moreover, the form of organization can vary, so next to business-oriented approaches - 4ocean, for example - one can find consortia like the Alliance to End Plastic Waste, a not-for-profit organization working in partnership with several multinational companies linked to the plastic value chain.

If these initiatives are successful, and we all hope so, the amount of collected marine litter is expected to be fairly large. But this is just a small part of the globally produced plastic trash - the tip of the iceberg. One should also consider the plastic reclaimed from municipal solid waste, which is even larger in volume and is usually destined to end up in dumps or landfills. What to do with all this garbage? Can we make good use of it as raw material? Conventional recycling strategies based on mechanical methods or incineration are downcycling approaches: they usually lead to products with lower values than the corresponding original plastic (often low-quality plastic) or inefficient energy recovery in the form of heat ${ }^{3}$. However, plastic waste can be seen as an important feedstock for the preparation of value-added materials, and here catalysis enters the scene.

\section{"However, plastic waste can \\ be seen as an important} feedstock for the preparation of value-added materials, and here catalysis enters the scene."

Catalytic recycling of plastic is not yet employed on a large scale, but has certainly shown great promise. Thermal treatment of reclaimed polyethylene terephthalate (PET) in the presence of a commercial guanidine catalyst and excess amounts of ethylene glycol, for instance, leads to the recovery of

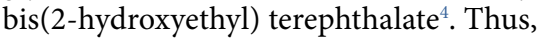
such a chemical can be newly employed for the preparation of high-quality PET. Clearly, the nature of the catalytic process depends on the chemical properties of the plastic to be transformed. Therefore, approaches that are closer to the refining of crude oil may be necessary in the case of more inert macromolecules, like for instance polyolefins. When conducted in a non-selective fashion, such processes can afford a mixture of hydrocarbons that can find application as alternative fuels. However, increasing the degree of selectivity, value-added chemicals can be targeted. A recent example has shown for instance the possibility of using hydrogenolysis to convert high-molecularweight polyethylene - typically found in single use plastic bags - into liquid hydrocarbons with a narrow molecular weight distribution, which can be applied as lubricants and waxes ${ }^{5}$. In this case, the desired selectivity could be achieved by tuning the structures of Pt nanoparticles supported on $\mathrm{SrTiO}_{3}$.

Plastic conversion is not a restricted area of research, if one looks at the diversity of possible approaches. Besides the heterogeneous or organocatalytic methods mentioned above, biocatalysis is also contending for its place. Enzymes, in fact, have shown very interesting activity in degrading plastics. Recently, Japanese scientists were able to isolate a specific bacterium from a microbial community localized in the vicinity of a PET recycling plant that features two enzymes competent for the hydrolysis of PET and its related intermediate - mono(2-hydroxyethyl) terephthalic acid - respectively ${ }^{6}$. Such families of enzymes are expected to play an important role in the development of practical upcycling schemes in the future.

The sceptical reader may at this point think that plastic reclaiming, recycling and upcycling has little hope due to economic constrains, especially in light of the current use once and discard paradigm. However, feasibility studies have shown that even employing existing technologies - that is, mechanical recycling and standard catalytic pyrolysis - the economic gain may be significant. McKinsey has recently published a study suggesting margins of around US\$60 billion for the plastic and petrochemicals sector if recycling based on existing technologies would be applied to 50 per cent of the plastics worldwide incidentally, no more than $12 \%$ is currently valorized.

Developing advanced and moreefficient catalytic conversion schemes for the upcycling of plastic waste is therefore an exciting opportunity for academic practitioners, and it is expected to further increase the profitability of the sector. Building blocks, monomers, and fine chemicals obtained in this manner can then be supplied to the polymer and fine chemical industry, according to the circular 
economy principle. At Nature Catalysis, we look with excitement at the future of this area, where catalysis once again has the possibility to contribute to a more sustainable future.
Published online: 14 November 2019 https://doi.org/10.1038/s41929-019-0391-7

References

$\square$ 1. Jambeck, J. R. et al. Science 347, 768-771 (2015).
2. Lebreton, L., Egger, M. \& Slat, B. Sci. Rep. 9, 12922 (2019).

3. Rahimi, A. \& García, J. M. Nat. Rev. Chem. 1, 46 (2017).

4. Fukushima, K. et al. J. Polym. Sci. A 49, 1273-1281 (2011).

5. Celik, G. et al. ACS Cent. Sci. https://doi.org/10.1021/ acscentsci.9b00722 (2019)

6. Yoshida, S. et al. Science 351, 1196-1199 (2016). 